\title{
Hybrid mastopexy: improving outcomes on implant-based augmentation mastopexy with fat
}

\author{
Giuseppe Cuccia ${ }^{1} \cdot$ Carola Maria Gagliardo ${ }^{2} \cdot$ Marco Romeo $^{3} \cdot$ Benedetto Di Trapani $^{4}$
}

Received: 21 December 2020 / Accepted: 12 April 2021 / Published online: 29 June 2021

(c) The Author(s) 2021, corrected publication 2021

\begin{abstract}
Background Autologous fat transplantation for breast augmentation represents an increasingly interesting technique in plastic surgery. Only a few standardized procedures are available. Hybrid augmentation mastopexy combines the benefits of autologous fat transplantation and implant-based breast augmentation mastopexy, reducing implant-related complications and prothesis size. Herein, we describe our surgical approach as a "hybrid aesthetic surgery."

Methods A retrospective analysis of all patients who underwent hybrid breast augmentation and lifting with simultaneous fat grafting was carried out. Clinical outcomes, ultrasound evaluation of upper pole fullness, aesthetic postoperative results, and complications were examined.

Results Eighteen patients with a mean age of 33 years (range: 24-52 years) and mean BMI of $25.8 \mathrm{~kg} / \mathrm{m} 2$ (range: 21.4-32.1 $\mathrm{kg} / \mathrm{m} 2$ ) were included in this study. Mean injected fat volume per breast was $115 \mathrm{cc}$ (range: $78-144 \mathrm{cc}$ ). Patients were followed up for a mean of 9.4 months (range: 6-24 months). No fat necrosis or major complications were encountered during the follow-up. Patient satisfaction was high in terms of breast shape, size, and coverage of the breast implant. No recurrence of ptosis was observed yet and no secondary revision surgery was performed.

Conclusions Hybrid mastopexy augmentation is an effective and safe procedure that combines the benefits of autologous fat grafting and implant-based breast augmentation. The transfer of autologous soft tissue allows obtaining a natural breast shape, reducing the onset of rippling and prothesis size. The reduction of prothesis size prevents ptosis recurrence but provides the desired projection. This reliable option improves long-term breast shape with elevated patient's satisfaction.
\end{abstract}

Level of evidence: Level IV, therapeutic study.

Keywords Mastopexy $\cdot$ Implant-based breast augmentation $\cdot$ Fat grafting $\cdot$ Plastic surgery

Presented in part during the $5^{\mathrm{TH}}$ Emirates Plastic Surgery

Congress, January 2020 Dubai, UAE

Carola Maria Gagliardo

carola.gagliardo@libero.it

1 Plastic Surgery Unit, Fatebenefratelli Hospital, Palermo, Italy

2 University Hospital Policlinico Paolo Giaccone, Palermo, Italy

3 Plastic Surgeon, Private Practice, Madrid, Spain

4 Surgeon Chef, Unit of General Surgery, Casa di Cura Torina, Palermo, Italy

\section{Introduction}

Mastopexy is a plastic surgery technique that consists of removing excess skin in ptotic breasts together with remodeling and lifting of breast parenchyma. It achieves several goals: breast and nipple areola complex elevation, conversion of breast shape from ptotic to a conical, volume increase/enhancement, good long-term results, and fewer complications. In the last decade, simultaneous augmentation mastopexy has been widely used. The standard procedure consists of an implant-based breast augmentation to restore or increase breast volume simultaneously with mastopexy. However, it continues to be debated because of the high rate of complications, such as unsatisfactory results, increased risk of implant infection with either exposure or malposition, increased risk of nipple or flap necrosis with potentially worse scars and decreased nipple sensation, 
capsular contracture, and palpable and visible implant [1, 2]. These complications are directly related to the size of the implant, which is used to better correct breast imprint and position on the chest wall.

Overcorrection of breast imprint may result in an aesthetically unpleasant shape of the breast together with stretching of the breast, atrophy of the gland, palpability, and visibility of the implant (rippling) [3, 4].

On the other side, autologous fat transplantation for breast augmentation has become an increasingly interesting surgical procedure but the technique is not yet standardized. Fat tissue transfer allows a natural breast shape to be obtained with good results and lower reoperation rate, often common in these patients [5, 6]. Fat necrosis remains another feared major complication but fortunately its incidence is decreasing more and more and outcomes are widely depending on the surgeon's expertise [7,8].

In the literature, several papers present a simultaneous breast augmentation based on implantation with mastopexy, while others describe a combined mastopexy and lipofilling. However, as far as we know, no retrospective or prospective studies on a hybrid approach between these methods have yet been described [9]. Precisely, starting from this scientific assumption, our retrospective study aims at describing our surgical technique "hybrid augmentation mastopexy," which combines an implant-based mastopexy and lifting with autologous fat transplantation, designed to achieve a natural result by analyzing its impact on the aesthetic result and patient satisfaction.

\section{Patients and methods}

This is a retrospective study on 18 consecutive female patients with a mean age of 33 years (range, 24 to 52 years) and mean body mass index (BMI) of $25.8 \mathrm{~kg} / \mathrm{m}^{2}$ (range, 21.4 to $32.1 \mathrm{~kg} / \mathrm{m}^{2}$ ) undergoing hybrid augmentation mastopexy. Inclusion criteria: informed patients undergoing this surgical approach. Patients were followed up at 1, 3, 6, 9, and 24 months. Clinical outcomes, ultrasound assessment of upper pole fullness, and postoperative results and complications were reviewed. The authors assessed aesthetic results using the physician-rated Global Aesthetic Improvement Scale (GAIS) and patient satisfaction using a verbal questionnaire. The degree of breast ptosis was between 2 and 3 points, according to "Regnault Classification" $[10,11]$. Our results have been evaluated and compared, respectively, with retrospective studies and meta-analyses found by searching on PubMed, Medline, Embase and Google Scholar, Web of Science, and the Cochrane Library.

\section{Surgical technique}

The markings for a circumvertical approach are executed locating the top of the periareolar region and deciding on the horizontal and vertical dimensions; the vertical component is added to the marking template. This is an important and relatively fixed landmark. The dimensions of the vertical sampling are entirely subject to intra-operative adjustment; and therefore, these initial vertical markings can be considered as preliminary.

The patient is placed supine on the operating table, under general anesthesia, with arms abducted on arm boards. After adequate preparation of the patient, the breast pocket is created by incorporating the 14-point plane through a central incision in the model [12]. A reusable implant sizer is inserted to assess the size of the pocket. This also provides the opportunity to assess and adjust (if necessary) the patient's pre-planned markings by custom skin fixation with the patient sitting upright.

We start locating the upper part of the areola at the distance of 18 to $20 \mathrm{~cm}$ from the midclavicular point. Then, we describe an ellipse shape that reduces the skin circle to a 42-mm diameter. Again, a manual pinch of the skin at the lower pole can provide a visual clue as to how much skin would be approximately removed to narrow the base diameter of the breast appropriately, and to take up the excess skin in the lower pole. Although this vertical sampling may be positioned slightly medially or laterally in some patients, it is generally ideal to base it directly in the meridian line of the breast. This will ensure that a roughly equal amount of breast skin will be removed both medially and laterally to enhance the creation of a

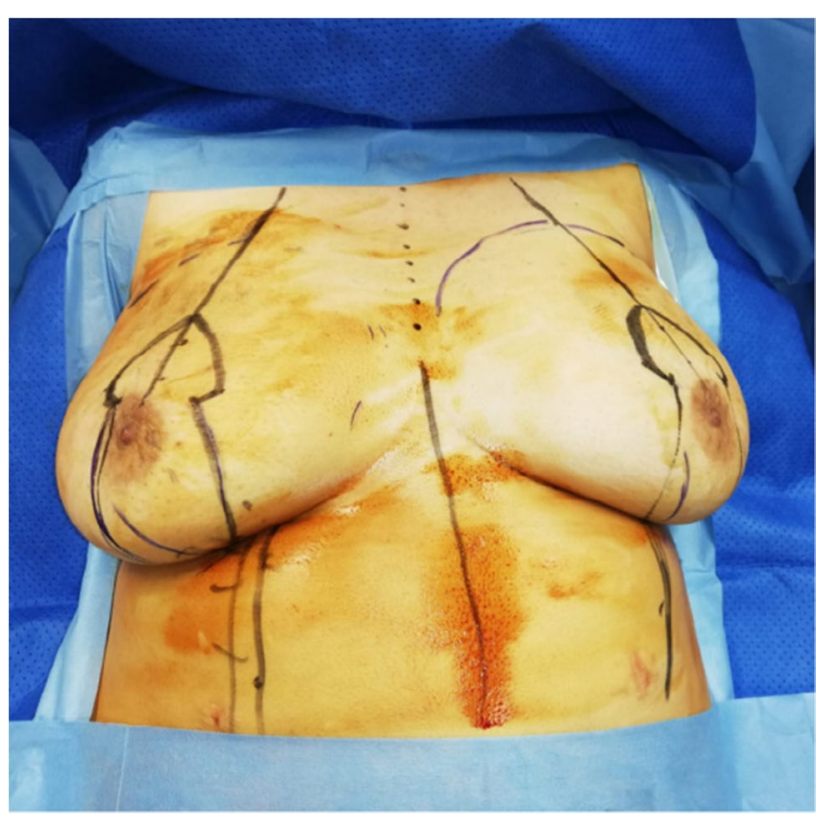

Fig. 1 Markings in hybrid mastopexy 
symmetrical breast profile. In smaller breasts, the vertical takeout extends directly down to the infra-mammary fold (IMF) but not beyond. It is actually advantageous to end the vertical resection just above the proposed IMF (between 2 and $3 \mathrm{~cm}$ ) as there is a general tendency of the lower breast pole to slide down onto the chest wall due to the weight of the prothesis. So, the exact dimensions of the model are fixed during surgery (Fig. 1).

Combined general anesthesia is chosen for all patients. Surgery usually starts infiltrating adrenaline $(1: 500,000)$ into the donor sites for fat harvesting, in the skin, and the lower quadrants to dissect them.

The main areas of the donor site (flank, abdomen, thigh, and knee) are infiltrated with Klein modified solution (Ringer's lactate, lidocaine $2 \%$, adrenaline $1 \%-1: 100,000)$. The tumescent technique, introduced by Klein, presents a large infiltrate volume with a ratio of infiltrate volume to total aspirated volume of $2-3: 1$.

Multi-punched 2-3-mm diameter cannulas, both 170-mm long with a rounded tip, help reduce pressure on each hole, decreasing damage in the samples collected. The 2-mm cannula has 5 round ports arranged in a spiral, each $1-\mathrm{mm}$ diameter, and the $3-\mathrm{mm}$ cannula has a single $3 \times 9 \mathrm{~mm}$ port sideways positioned. The $2-\mathrm{mm}$ cannula concurrently facilitates harvesting from more superficial and vascularized layers of adipose tissue, and reduces patient discomfort and trauma.

The collected fat solution is prepared for injection and it is left for decantation during the mastopexy. The areola is marked with a breast cookie-cutter of $42-\mathrm{mm}$ diameter.

Strategically, we start with a vertical incision right in the lower quadrant of the marked model to first place the implant sizer and then re-tailor the skin envelope around the new breast volume.

This approach will prevent excessive reduction of the skin envelope from hindering the easy placement of an appropriately sized implant. The procedure begins by making an incision in the center of the previously marked vertical resection area and the underside of the breast is exposed. It is important to stay above Scarpa's fascia as it inserts into the base of the breast as the pocket is developed to avoid opening up the loose subscarpal space below the fold. In this way, the anatomical integrity of the fold will be maintained and the likelihood of inadvertently lowering the fold will be reduced. From this point of access, a subglandular or a subpectoral pocket can be easily obtained; $90 \%$ of cases treated by the senior author are subglandular and subfascial pockets.

To determine the implant size, the width of the breast is measured, and one-half of the medial breast pinch thickness was subtracted from the width of the base to yield the optimal implant base.

Once the implant size is established, the variation of the implant projection options determined the final volume, which is discussed previously between the patient and the surgeon according to the patient's recommendations and wishes; ultimately, the amount of transplanted fat is decided following upper pole pinch thickness (range 75-144 cc per breast).

In order to apply the model, a good reproducible sequence consists of stapling the areola into the defect and then plicating the lower pole as necessary to create an attractive and rounded lower pole contour. Once the final breast volume
Fig. 2 Tailor taking approach of mastopexy with sizer and skin deepithelization
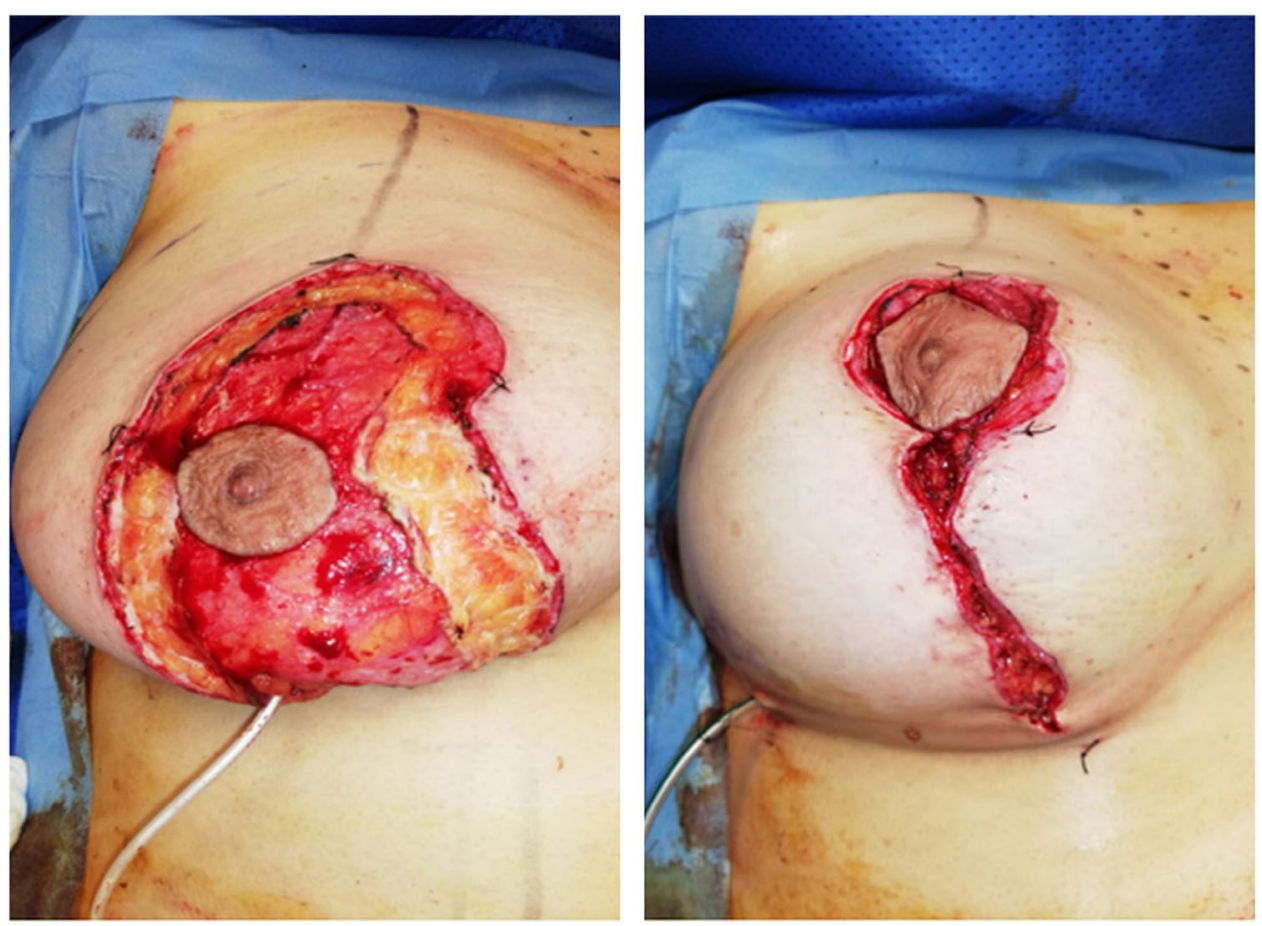
and shape have been determined, the vertical plication line is marked with a surgical pen (Fig. 2).

The implant is inserted and properly positioned; the breast parenchyma is accosted along the vertical incision by closing the medial and lateral parenchymal pillars with both deep and superficial sutures, using resorbable stitches. The average implant size is $285 \mathrm{cc}$ (range $255-360 \mathrm{cc}$ ). The implant used were round gel-filled macro- and microtextured Polythec (®Germany) implants. Fifteen patients received high-profile implants, while remining patients moderateprofile ones. The vertical skin incision is closed by layers, again with a layer of interrupted and running absorbable 3-0 sutures.

At the end of the augmentation mastopexy procedure, we use fat grafting as described below.

We use fat mainly in the upper quadrants because of poor results in a long-term follow-up of augmentation mastopexy.

The markings for the autogenous fat grafting area are made temporarily in the cleavage limits, which represent the future transition between the implant and non-implant zones in order to achieve a homogeneous transition.

A small stab incision (18-Gauge needle) is performed in the upper lateral part of the upper quadrant bilaterally, along the anterior axillary line and on the medial part of the inframammary fold. Through these incisions, we graft the fat previously harvested. The mean injected fat volume was $115 \mathrm{cc}$ (range, 75-144 cc) per breast (Figs. 3 and 4). After dressing placement, the patient will have to wear a supportive compression bra for 6 weeks.

\section{Subglandular/subfascial vs submuscular placement}

Which of the two types of prosthetic implant site (subfascial or submuscular placement) is more adequate remains a theme of discussion among surgeons, concerning the

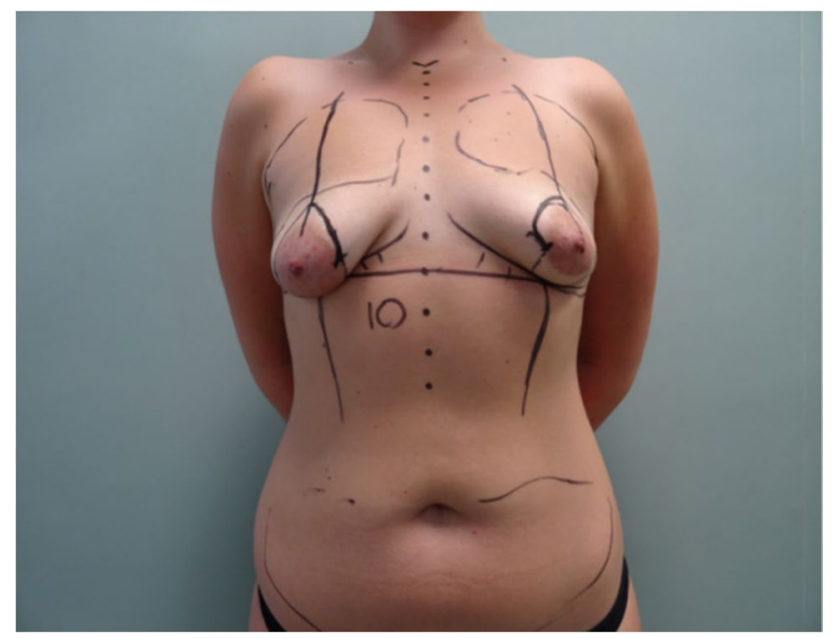

Fig. 3 Schematic view of marked patient and upper pole fat grafting

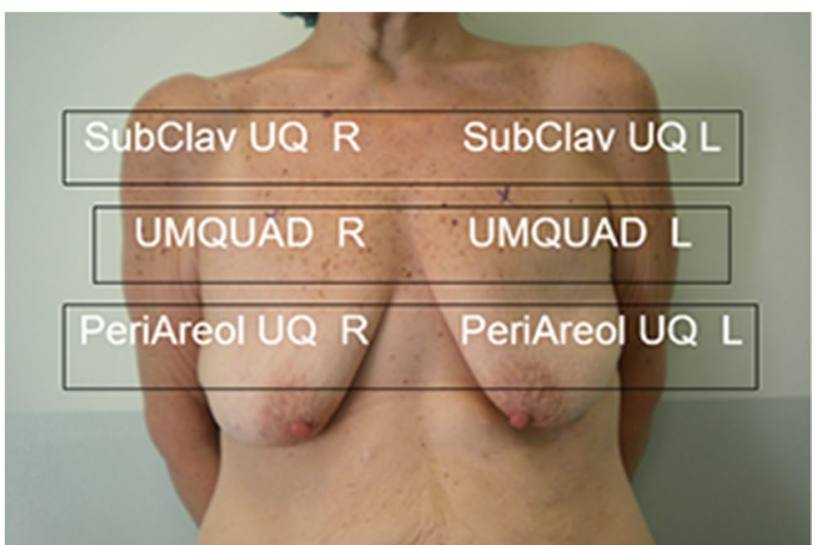

Fig. 4 Schematic view of fat distribution on the upper quadrant: SubClav UQ R, subclavicular upper quadrant right; SubClav UQ L, subclavicular upper quadrant left; UMQUAD R, upper medial quadrant right; UMQUAD L, upper medial quadrant left; Periareolar UQR, periareolar upper quadrant right; Periareolar UQL, periareolar upper quadrant left

appropriate coverage for the implant, rippling, contracture, animation, and aesthetic outcomes.

Whereas there is no definite agreement about the definitive pocket, surgeons advocate satisfactory results according to each own's practice. Generally, submuscular pocket allows a lower grade of contracture and a better implant concealing, but runs higher risk of animation, cephalic, and/or lateral displacement and waterfall effect in the long term [13].

On the other hand, subglandular/subfascial pocket has higher risk of contracture, double capsule, and visible rippling but provides almost a painless surgery, and a natural and stable look with the implant well coupled with the gland in the long run [14].

About our experience with the employment of both types of procedure, we mitigate the side effect through perioperative measures and details. The submuscular pocket requires a complete release of muscle in the lower quadrants, including lower sternal fibers, with or without perpendicular split, creating rather a dual-plane surgery; lateral wall support is also mandatory.

Subglandular positioning requires a tight pocket, a low-textured implant, and good external support (casting) to avoid implant floating to prevent double capsule formation.

Finally, patient selection is a key point to determine the best plane according to skin quality, muscle hypertrophy, and tissues' thickness to provide implant coverage, support, and reduce the risk of rippling.

In our patients, in both cases, lipofilling has provided a controllable extra layer to minimize these overmentioned risks. 

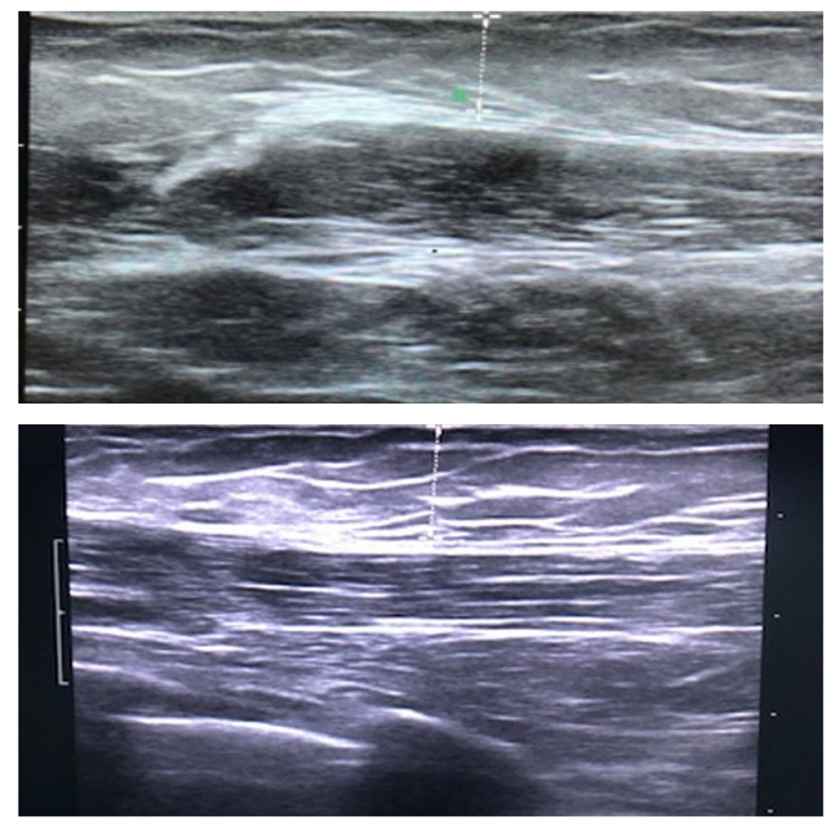

Fig. 5 Ultrasound evaluation of upper pole fullness and the fat retention rate pre-operatively (upper part of the figure) and 6 months postoperatively (lower part of the figure)

\section{Ultrasound evaluation}

The ultrasound evaluation of upper pole fullness and the fat retention rate were examined preoperatively and 6 months postoperatively. For practical/schematic reasons, the authors divided the upper quadrants into three different areas where subcutaneous fat tissue was detected: subclavicular, medial, and inferior (periareolar) upper quadrants bilaterally (Fig. 5).

\section{Results}

No major complications such as fat necrosis, skin or nippleareola complex necrosis, capsular contracture, decreased nipple sensation, implant extrusion, re-operation rate for asymmetry o unsatisfactory results, and scar revisions were encountered during a mean follow-up time of 9.4 months (range, 6 to 24 months). Our findings show optimal aesthetic outcomes and an elevated patient satisfaction in terms of breast shape, size, and coverage of the breast implant. Patient satisfaction was evaluated from the chart at the most recent follow-up and similar results were found considering the aesthetic outcomes of the upper quadrant using the physician-assessed Global Aesthetic Improvement Scale (GAIS - from 1 to 5). A total of $90 \%$ of patients scored degree 1 (excellent corrective result) and 10\% degree 2 (marked improvement of the appearance, but not completely optimal) GAIS score.
Ptosis was corrected and volume desired was achieved in all patients. No recurrence of ptosis-at least 2 years follow-up-has been observed yet and no secondary correcting surgery has been performed. Two patients had minor complications such as delayed wound healing in the lower quadrants and at the junction of areola and vertical scar, which required no surgical revision. Another patient developed a hypertrophic scar which was successfully treated with silicon taping and gel dressing.

Both ultrasound assessment and aesthetic physician panel score showed that, in the three different parts of the upper quadrant, a higher fat retention rate was found in the subclavicular area, if compared to the medial and periareolar parts of the upper quadrant.

The mean measure of subcutaneous thickness in this area was $10.8 \mathrm{~mm}$ (pre-operatively mean value: $6.59 \mathrm{~mm}$ ) with an increase of $39 \%$ of subcutaneous tissue in the subclavicular area and an average of fat retention rate after injection between 60 and $70 \%$.

Patient satisfaction was evaluated during ultrasound follow-up by the two senior surgeons (plastic and breast surgeons), using an acquired verbal and informal questionnaire to grade the patient's level of satisfaction with the aesthetic results. Postoperative photographs were obtained at followup appointments and compared with the pre-operative images.

Representative cases are presented in Figs. 6, 7, and 8.

\section{Discussion}

Augmentation mastopexy is an aesthetic breast surgery procedure that seeks to create youthful, beautiful-appearing breasts wherein age, hormonal changes, or weight loss had led to alterations that require intervention on the skin envelope and breast parenchyma [11].

As reported in most current studies, complications of mastopexy with risk of recurrent ptosis are implant-related. This is due to the incision, the implant placement pocket, and implant size and weight [15]. Moreover, the singlestage augmentation mastopexy is increasingly associated with demands for larger implants to improve projection and fullness of the upper pole.

The novelty of our procedure consists of a hybrid augmentation mastopexy, combining the implant-based breast augmentation and lifting with autologous fat grafting. In fact, the employment of an implant provides the desired projection and fullness of the upper pole, while autologous soft tissue allows obtaining a natural breast shape with reduced implant-related problems [5, 11].

In general, in relation to the implant-based augmentation mastopexy, major complications include a high rate of re-intervention for asymmetry, waterfall deformity or 
Fig. 6 a, b A 35-year old woman with a grade III of ptosis, treated with hybrid mastopexy: $335 \mathrm{cc}$ round implant and $95 \mathrm{cc}$ fat bilaterally. c, d Postoperative results at 24 months follow-up
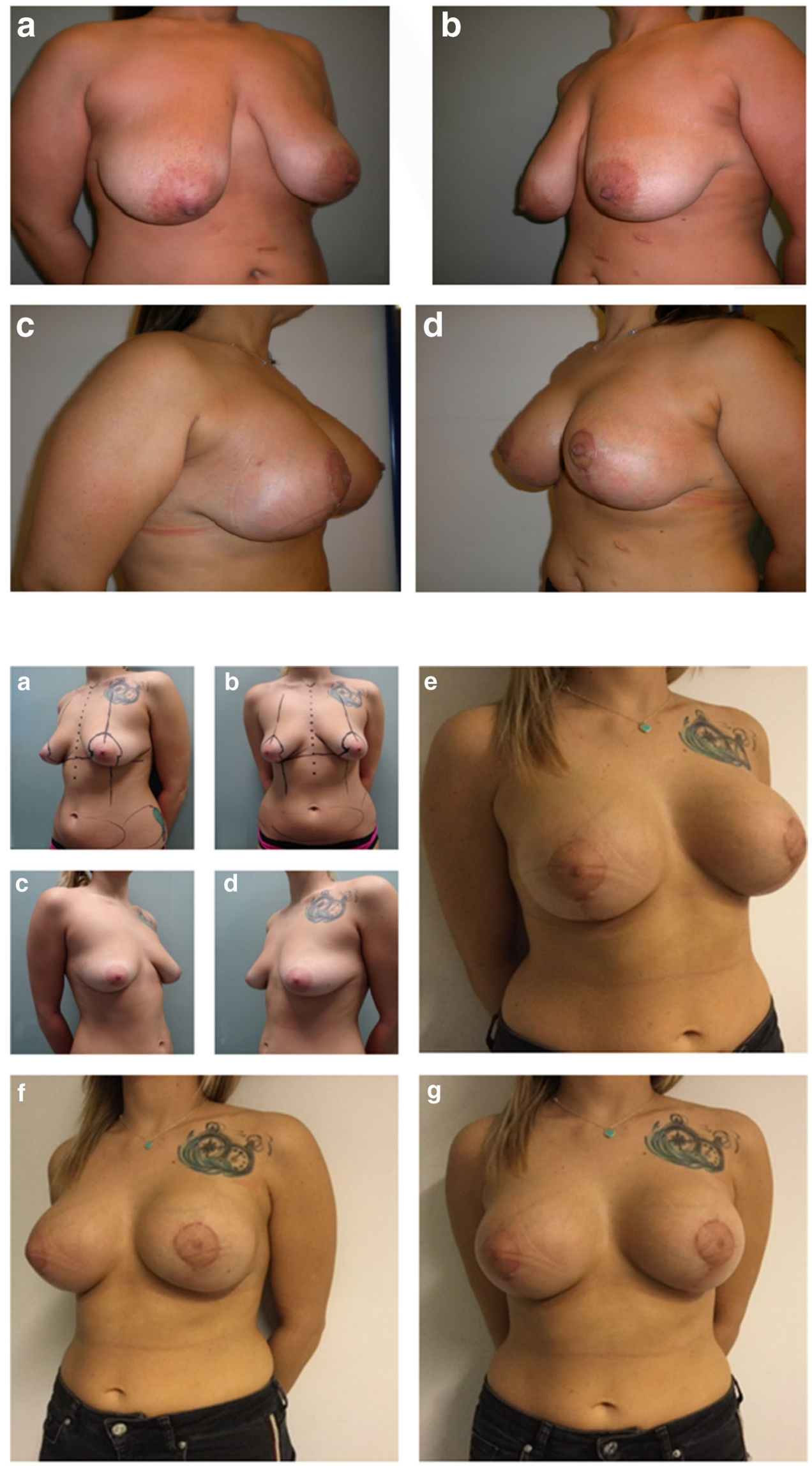
Fig. 8 a, b A 48 year-old patient with a grade III of ptosis and Baker IV capsular contracture. Anchor-type mastopexy with $105 \mathrm{cc}$ fat bilaterally. c, d Postoperative views at 18 months follow-up
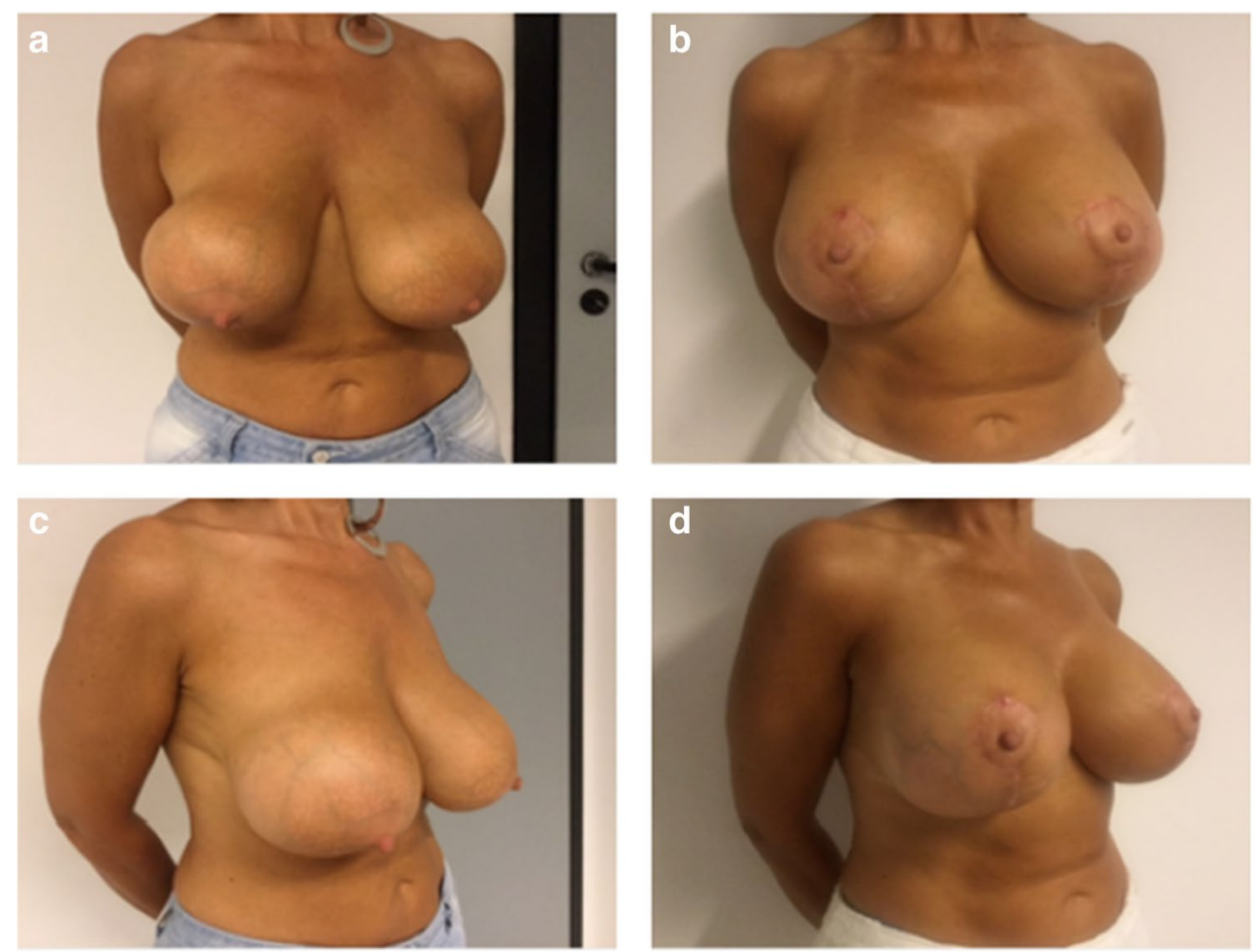

unsatisfactory results (15-23\% of cases), skin or nippleareola complex necrosis, capsular contracture, rippling, decreased nipple sensation, implant extrusion, and scar revisions [6]. Fat necrosis is a frequent complication as a concern of fat transplantation [16-18].

Our patients were followed up at 1, 6, 9, and 24 months, during which these clinical outcomes, ultrasound evaluation of upper pole fullness, and postoperative results and complications were examined. Performing our hybrid surgical techniques, we have not noticed any major complications, while we experienced few minor complications such as delayed wound healing. We recorded a high degree of satisfaction in terms of desired fullness and long-term shape.

In literature, several surgical techniques are described improving the classical isolated mastopexy, with an overall complication rate of $13-36 \%$. On the contrary, the main purpose of our work is to point out that, as far as we know, our hybrid approach to mastopexy, moreover without major complications, is not mentioned in any work [19-21].

In this difficult type of surgery, a main advantage of adipose tissue consists of allowing us to reduce the implant size. Moreover, a smaller implant size strongly prevents the occurrence of ptosis and the other implantrelated complications, such as capsular contracture, rippling, implant palpability and visibility, animation muscle deformity, and waterfall deformity (related to sub-muscular implant position) [22-25]. As proof of this, in our single-center experience, no recurrence of ptosis has yet been observed and no secondary correcting surgery has been performed. In literature, this ranges from $36 \%$ at 10 years $[6,26]$.

In recent studies, in fact, fat transplantation has been used as an additional treatment to currently used therapies to treat capsular contracture, with satisfactory cosmetic results and excellent pain management. This could probably also be due to the contrasting anti-inflammatory role of the adipose tissue $[27,28]$. The implant is placed in the deepest part of the breast and represents the supporting core, around which the adipose tissue is used to sculpt the shape of the breast.

In our experience, we have performed $90 \%$ of cases with subfascial implant position that allows applying this hybrid breast surgery to thin patients as well.

This technique can also be applied to women of different ages and/or degrees of ptosis; the ptosis is corrected; the volume is enlarged/expanded. We did not use any software analysis to plan the amount of fat injection because we refer to upper pole pinch test to establish the amount of fat grafting and then we analyzed the amount of fat taken during ultrasound follow-up to confirm our results [29].

Finally, these excellent results, compared with those in literature, have encouraged us to divulge our innovative hybrid approach and to continue performing the technique in women of various ages and/or degrees of ptosis who desire both shape and volume correction. We emphasize that achieving optimal results requires more than just an adequate technique: great aesthetic results depend on the expertise of the surgeon. 


\section{Conclusions}

Hybrid mastopexy augmentation is an effective and safe procedure that combines the advantages of autologous fat transplantation and implant-based breast augmentation. The implant provides the desired projection and fullness of the upper pole, while the transfer of soft tissue allows a natural breast shape with moderate scarring, without complications and secondary corrective surgery. Patient's satisfaction is high. This reliable option improves long-term breast shape and it avoids recurrence of ptosis. Finally, these excellent results encourage us to continue performing the technique in women of varying ages and/or degrees of ptosis who desire both shape and volume correction. However, we advise female population to consult professional help only because great results depend on the expertise of the surgeon. Finally, this is a retrospective study so other prospective works are needed for a better evaluation of our hybrid surgical procedure.

Data availability Data have been collected with transparency and without distortions.

\section{Declarations}

Ethics approval All procedures performed in studies involving human participants were in accordance with the ethical standards of the institutional and/or national research committee and with the 1964 Helsinki Declaration and its later amendments or comparable ethical standards. This is retrospective study. A local research ethics committee has confirmed that no ethical approval is required.

Informed consent Informed consent was obtained from all individual participants included in the study.

Patient consent Patients signed informed consent regarding publishing their data and photographs.

Conflict of interest Giuseppe Cuccia, Carola Maria Gagliardo, Marco Romeo and Benedetto Di Trapani declare no conflict of interest.

Funding Open access funding provided by Università degli Studi di Palermo within the CRUI-CARE Agreement.

Open Access This article is licensed under a Creative Commons Attribution 4.0 International License, which permits use, sharing, adaptation, distribution and reproduction in any medium or format, as long as you give appropriate credit to the original author(s) and the source, provide a link to the Creative Commons licence, and indicate if changes were made. The images or other third party material in this article are included in the article's Creative Commons licence, unless indicated otherwise in a credit line to the material. If material is not included in the article's Creative Commons licence and your intended use is not permitted by statutory regulation or exceeds the permitted use, you will need to obtain permission directly from the copyright holder. To view a copy of this licence, visit http://creativecommons.org/licenses/by/4.0/.

\section{References}

1. Jourdain DA, Oren T, Steven C, Shukan P, Radbeh T, Michael M (2019) Can it be safe and aesthetic? An eight-year retrospective review of mastopexy with concurrent breast augmentation. Plast Reconstr Surg Glob Open 7(6):e2272

2. Eisenberg T (2012) Simultaneous augmentation mastopexy: a technique for maximum en bloc skin resection using the inverted-T pattern regardless of implant size, asymmetry, or ptosis. Aesthetic Plast Surg 36(2):349-354

3. Spear SL, Dayan JH, Clemens MW (2009) Augmentation mastopexy. Clin Plast Surg 36:105-115

4. Rohrich RJ, Gosman AA, Brown SA et al (2006) Mastopexy preferences: a survey of board-certified plastic surgeons. Plast Reconstr Surg 118:1631-1638

5. Kwiatkowska K, Krapohl BD, Tanzella U, Ueberreiter K (2019) Long-term clinical results and quality of life in patients undergoing autologous fat transplantation for breast augmentation using the BEAULI ${ }^{\mathrm{TM}}$ protocol. GMS Interdiscip Plast Reconstr Surg DGPW 8:Doc10

6. Calobrace MB, Herdt DR, Cothron KJ (2013) Simultaneous augmentation/mastopexy: a retrospective 5-year review of 332 consecutive cases. Plast Reconstr Surg 131(1):145-156

7. Al Sufyani MA, Hargan AH, Shammari NA, Al Sufyani MA (2016) Autologous fat transfer for breast augmentation: a review. Dermatol Surg 42(11):1235-1242

8. Guo X, Jin X (2019) Management of fat necrosis after autologous fat transplantation for breast augmentation. Plast Reconstr Surg 144(4):700e-701e

9. Graf RM, Closs Ono MC, Pace D, Balbinot P, Pazio ALB, de Paula DR (2019) Breast auto-augmentation (mastopexy and lipofilling): an option for quitting breast implants. Aesthetic Plast Surg 43(5):1133-1141

10. Regnault P (1976) Breast ptosis. Definition and treatment Clin Plast Surg 3(2):193-20311

11. Qureshi AA, Myckatyn TM, Tenenbaum MM (2018) Mastopexy and mastopexy-augmentation. Aesthet Surg J 38(4):374-384

12. Adams WP Jr, Culbertson EJ, Deva AK et al (2017) Macrotextured breast implants with de ned steps to minimize bacterial contamination around the device: experience in 42,000 implants. Plast Reconstr Surg 140(3):427-431

13. Weck Roxo AC, Xerfan Nahas F, Salin R, Cardoso de Castro C, Aboudib JH, Garcia Marques R (2016) Volumetric evaluation of the mammary gland and pectoralis major muscle following subglandular and submuscular breast augmentation. Plast Reconstr Surg 137(1):62-69

14. Brown T (2020) Plane change vs capsulotomy: a comparison of treatments for capsular contraction in breast augmentation using the subfascial plane. Aesthetic Plast Surg. https://doi.org/ 10.1007/s00266-020-02010-8

15. Khavanin N, Jordan SW, Rambachan A, Kim JYS (2014) A systematic review of single -stage augmentation-mastopexy. Plast Reconstr Surg 134:922

16. Manero I, Rodriguez-Vega A, Labanca T (2019) Combined breast reduction augmentation. Aesthetic Plast Surg 43(3):571-581

17. Doshier LJ, Eagan SL, Shock LA, Henry SL, Colbert SH, Puckett CL (2016) The subtleties of success in simultaneous augmentation-mastopexy. Plast Reconstr Surg 138(3):585-592

18. Li S, Chen L, Liu W, Mu D, Luan J (2018) Capsular contracture rate after breast augmentation with periareolar versus other two (inframammary and transaxillary) incisions: a meta-analysis. Aesthetic Plast Surg 42(1):32-37 
19. Asserson DB, Kulinich AG, Orbay H, Sahar DE (2019) Differences in complication rates of gluteoplasty procedures that utilize autologous fat grafting, implants, or local flaps. Ann Plast Surg 82(5S Suppl 4):S342-S344

20. Stevens WG, Macias LH, Spring M et al (2014) One-stage augmentation mastopexy: a review of 1192 simultaneous breast augmentation and mastopexy procedures in 615 consecutive patients. Aesthet Surg J 34:723-732

21. Hall-Findlay EJ (2011) Breast implant complication review: double capsules and late seromas. Plast Reconstr Surg 127:56-66

22. Hildago D, Spector J (2014) Breast augmentation. Plast Reconstr Surg 133:567e-583e

23. Coleman SR1, Saboeiro AP (2015) Primary breast augmentation with fat grafting. Clin Plast Surg 42(3):301-6, vii

24. De Lima E, Souza R, Apgaua BT, Milhomens JD, Albuquerque FT, Carneiro LA, Mendes MH et al (2016) Severe fat embolism in perioperative abdominal liposuction and fat grafting. Braz $\mathbf{J}$ Anesthesiol 66(3):324-328

25. Strong AL, Cederna PS, Rubin JP, Coleman SR, Levi B (2015) The current state of fat grafting: a review of harvesting, processing, and injection techniques. Plast Reconstr Surg 136(4):897-912
26. Wang CL, Luan SS, Panayi AC, Xin MQ, Luan J (2019) Methods used for evaluation of volume retention rate in autologous fat grafting for breast augmentation: a systematic review. Chin Med J (Engl) 132(18):2223-2228

27. Papadopoulos S, Vidovic G, Neid M, Abdallah A (2018) Using fat grafting to treat breast implant capsular contracture. Plast Reconstr Surg Glob Open 6(11):e1969

28. Smitka K, Marešová D (2015) Adipose tissue as an endocrine organ: an update on pro-inflammatory and anti-inflammatory microenvironment. Prague Med Rep 116(2):87-111

29. João M, Mendonça Munhoz A, Pedron M, Pinto de Oliveira AC, Duarte DW, Neto R et al (2020) Hybrid breast augmentation: a reliable formula for preoperative assessment of fat graft volume based on implant volume and projection. Aesthetic Surg J 40(8):NP438-NP452

Publisher's Note Springer Nature remains neutral with regard to jurisdictional claims in published maps and institutional affiliations. 